\title{
Challenging issues in molecular-targeted therapy
}

\author{
Zoheir Ezziane \\ Applied Science and Technology, \\ Higher Colleges of Technology, \\ Al Ain, United Arab Emirates
}

Correspondence: Zoheir Ezziane Applied Science and Technology, Higher Colleges of Technology, PO Box 17258 Al Ain, United Arab Emirates Tel +97 I37095 229

Fax +97 I37 820766 Email zoheir.ezziane@hct.ac.ae

\begin{abstract}
There are variety of anticancer treatments including chemotherapeutic drugs, which are known to induce cell growth arrest and apoptosis through DNA damage and cytoskeleton toxicity. Meanwhile, histone deacetylase (HDAC) inhibitors could apply their antitumor activity through chromatin remodeling and gene expression modulation that affect the cell cycle and survival pathways. This paper proposes an anticancer three-drug compound and discusses several challenging issues in relation to designing multidrug compounds that could possibly lead to molecular-targeted therapies.
\end{abstract}

Keywords: molecular targeted therapy, combination therapy, epigenetic therapy, anticancer drug, personalized medicine product

\section{Introduction}

There is accurate machinery that allows cells to recognize themselves and undertake specific tasks. This machinery represents the blueprint of various patterns of gene activation/inactivation throughout the cell cycle. It is mediated by numerous processes such as DNA methylation, adenosine triphosphate (ATP)-dependent chromatin remodeling, and post-translational modifications of histones, which include the acetylation and deacetylation of amino groups of lysine residues present in histone N-terminal tails. The set of chromatin modifying enzymes include one of the histone modification enzymes, such as histone acetyltransferase (HAT), histone deacetylase (HDAC), histone methyltransferase (HMT), histone demethylase (HDMT), and histone kinase. HATs act as transcriptional coactivators, whereas HDACs act as transcriptional corepressor enzymes. Lack of expression or repression leads to an irregular outcome for the cell, including altered genetic programs and increased rate of cell transformation.

A number of silenced tumor suppressor genes are shown to be lost due to epigenetic inactivation rather than sequence damages, although, epigenetic changes cooperate with genetic changes to initiate the development of a cancer since they are mitotically heritable. ${ }^{1,2}$ Further, epigenetic irregularities are pharmacologically reversible as oppose to genomic damage. ${ }^{3}$ This fact provides an incentive to devote more efforts in designing combination drugs that eventually achieve epigenetic and molecular-targeted therapies.

\section{Combination therapy}

It is known that the whole epidermal growth factor receptor (EGFR) family of proteins contributes to tumor cell growth in numerous types of cancer. When activated by particular molecules that bind to their ligands, such receptors transmit a proliferation signal to the cell by initiating a cascade of internal molecular interactions. Cancer cells also secrete a variety of growth factors to attract the endothelial cells that build new blood vessels (ie, angiogenesis), which subsequently make tumors more difficult to treat.

The tangle of signaling pathways leading from the receptors to the cellular processes that actually cause a cell to divide or to resist suicide despite DNA damage is highly complex. As depicted in Figure 1, a potential drug compound may suppress the 
growth of cancer cells by inducing either G1 or G2 arrest, DNA synthesis, apoptosis, and should have antitumor activity against various types of cancer. Synergistic inhibition of cell proliferation and induction of apoptosis is an important factor towards effective combination therapy.

A combination between HDAC inhibitors like NVPLAQ824 and vascular endothelial growth factor (VEGF) receptor 2 kinase inhibitors like PTK787/ZK222584 has been demonstrated to have an antiangiogenic and antitumor effect. ${ }^{4}$ This drug combination resulted in a greater antitumor and antiangiogenic effect in vitro and in vivo compared with single agents (ie, monotherapies).

Many HDAC inhibitors mediate their antitumor activity by chromatin remodeling and then followed by gene expression modulation. The antiproliferative effect of an HDAC inhibitor on tumor cells is supposed to correlate with the induction of a specific gene expression that mediate cell cycle arrest and/or apoptosis. In addition, HDAC inhibitors may also repress gene expression by inducing protein acetylation. For example, induction of p21 and inhibition of survivin expression may compromise the proliferation and differentiation of endothelial cells. ${ }^{4}$

Mitogen-activated protein kinases (MAPK) and AKT (a serine/threonine kinase also known as protein kinase B) pathways play important roles in cell proliferation, ${ }^{5}$ and therefore a compound that induces apoptosis is also supposed to inhibit AKT and/or MAPK activation. In addition, MAPK plays a role of a signaling mediator of EGFR in blockade of apoptosis. This presumes a significant contribution of MAPK in cell survival, since it is also activated by EGF stimulation independent of epidermal receptor growth factor 2 (ErbB2) and epidermal receptor growth factor 3 (ErbB3) signaling in human colon cancer (GEO) cells. ${ }^{6}$ Unknown mechanisms or pathways that react to the compensatory activation of EGFR in response to the down-regulation of ErbB2 phosphorylation need to be explored. Hence, this study investigates the combination of an EGFR inhibitor with an HDAC inhibitor and subsequently analyze the corresponding level of inhibitory effects on ErbB2 phosphorylation. This combination therapy is discussed and analyzed later in this paper.

A drug's antitumor and antiangiogenesis effect needs to be correlated with the down-regulation of angiogenesisrelated genes such as VEGF and survivin. ${ }^{7,8}$ This type of drug also needs to modulate the expression of multiple genes that contribute in tumor development and angiogenesis, and it is required to induce other inhibition of VEGF signaling and angiogenesis as it is depicted in Figure 1. Further, the design of a potential compound with an antitumor effect must affect tumor growth by acting on independent and parallel pathways. A combination therapy should induce cell cycle arrest by gene expression modulation in epithelial tumor and endothelial cells. As a result, the newly designed drug has the potential to be tailored for individual patients. For instance, this therapy can target patients with tumors that are dependent upon VEGF, angiogenesis-related genes, EGFR, and ErbB2. This type of drug represents a potential molecular targeted therapy that is also called key personalized medicine product.

\section{Clinical trials of multidrug compounds}

During a phase I study, a combination of trabectedin and pegylated liposomal doxorubicin (PLD) was tested in 36 patients with advanced malignancies. ${ }^{9}$ An overall response rate of $16.7 \%$ was reported including one complete response (CR) and five partial responses (PR), and 39\% had

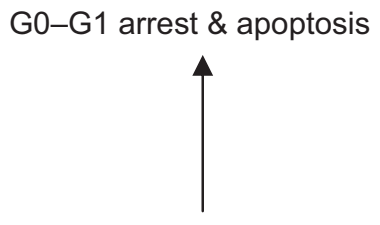

Tumor proliferation cells

\& VEGF

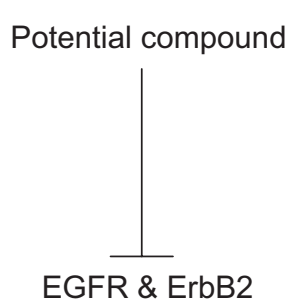

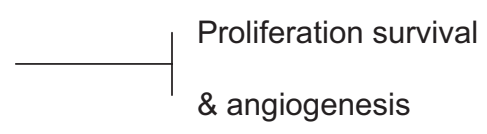

\& angiogenesis

Figure I Representation of concomitant molecular changes after combination treatment with a potential compound: Combination therapies with agents that target endothelial cells to block angiogenesis, EGFR/ERBB2, and histone deacetylase inhibitors to prevent tumor adaptation in cancer treatment warrants experimental studies.

Abbreviations: EGFR, epidermal growth factor receptor; ErbB2, epidermal receptor growth factor 2; VEGF, vascular endothelial growth factor. 
stable disease (SD). This study also confirmed that trabectedin combined with PLD provides a potential clinical advantage, and it is generally well tolerated at therapeutic doses in pretreated patients with various tumor types.

A combination of immunosuppressive agents cyclophosphamide (CYC) and imatinib was evaluated in five patients with advanced scleroderma-related interstitial lung disease. ${ }^{10}$ This combination was tolerated and without major effects in all patients. From the two patients who completed one year of treatment, only one patient with mild restrictive lung disease showed improvement in pulmonary function. A phase I/II study tested the combination of gefitinib and rofecoxib in 42 patients with nonsmall cell lung cancer. ${ }^{11}$ This study reported $2.3 \% \mathrm{CRs}$, $4.7 \% \mathrm{PRs}$, and $28.5 \%$ SDs. In addition, the treatment was also reported to be generally tolerated.

Thirty-one open-angle glaucoma patients who were insufficiently controlled on latanoprost monotherapy, were given dorzolamide/timolol (DTFC), latanoprost/timolol fixed combination (LTFC), or a combination of DTFC and latanoprost. ${ }^{12}$ This study showed that the latter therapy considerably decreased the intraocular pressure (IOP). In a similar study, a combination therapy of brimonidine and timodol was effective in decreasing the IOP as opposed to monotherapy with brimonidin or timodol. ${ }^{13}$

In a phase I study, a combination of DNA-hypomethylating agent (5-AZA) and an HDAC inhibitor (valproic acid) was assigned to 55 patients with advanced cancer. ${ }^{14}$ This report showed $25 \%$ SDs in which the disease stabilized from four to 12 months, and a considerable decrease in global DNA methylation and induction of histone acetylation were also observed. A phase II study of epigenetic therapy with hydralazine and magnesium valproate was reported. ${ }^{15}$ Seventeen patients were evaluable for toxicity and 15 for responses. Primary sites included cervix (3), breast (3), lung (1), testis (1), and ovarian (7) carcinomas. The results showed 26.7\% PRs and $53 \%$ SDs. The main toxicity was hematologic-related. Further, global DNA methylation, HDAC activity, and promoter demethylation were observed. A phase II trial was conducted to investigate clinical and molecular responses mediated by a histone deacetylase inhibitor (Depsipeptide FK228) in lung cancer patients. Nineteen patients were evaluable for toxicity and 18 for responses. This report showed neither significant cardiac toxicities nor objective responses, ${ }^{16}$ since small does were assigned. However, a combination with other compounds warrants further evaluation in lung cancer patients.

The initial phase I/II trial of bortezomib and melphalan in 35 patients, who had progressed on relapsed or refractory multiple myeloma (MM) was reported. ${ }^{17}$ Responses were observed in $68 \%$ of the studied population including $6 \%$ CRs, 9\% immunofixation-positive CRs, 32\% PRs, and 21\% minimal response (MR). The combination of bortezomib and melphalan was reported to have a controllable toxicity and represent a compelling therapy candidate for MM patients. In a later report, a phase I/II study was conducted to assess the combination of arsenic trioxide/bortezomib/ascorbic acid (ABC) in 22 patients who had progressed on MM. ${ }^{18}$ This study showed that responses were only observed in $27 \%$ of the heavily pretreated study population including 9\% PRs and $18 \%$ MRs. The ABC treatment was reported to be well tolerated by the majority of patients.

One thousand ninety-one patients with type 2 diabetes were treated with the combination therapy of sitagliptin and metformin. ${ }^{19}$ This regimen was reported tolerable amongst all patients and provided an additive glycemic improvements. Similar results were obtained when 217 type 2 diabetes patients were treated with the combination therapy of sulfonylurea and metformin. ${ }^{20}$

A novel farnesyl protein transferase inhibitor (BMS214662) combined with paclitaxel and carboplatin was assigned in 30 patients with solid tumors. ${ }^{21}$ The purpose of this study was to investigate the toxicities, pharmacokinetics, and pharmacodynamics of BMS-214662 when combined with the other two compounds. This report showed no particular pharmacokinetic interaction between BMS-214662 and paclitaxel, and the combination of BMS-214662 with paclitaxel and carboplatin was normally tolerated in solid tumors. This phase I trial also reported 3.3\% PRs related to a taxane-resistant esophageal cancer, 6.6\% PRs related to endometrial and ovarian cancer, and $26.6 \%$ SDs. A phase I study also investigated the combination of P-glycoprotein inhibitor (MS209) with docetaxel in patients with solid tumors. ${ }^{22}$ This study showed the absence of strong pharmacokinetic interaction between the two compounds at moderate dose levels, and the combination therapy had a limited effect on docetaxel toxicity or pharmacokinetics.

In a phase Ib study, the combination of patupilone and carboplatin was tested in 26 patients with recurrent platinumsensitive ovarian cancer. ${ }^{23}$ Tumor response was assessable by response evaluation criteria in solid tumors in 17 patients including 6\% CRs and 59\% PRs. This report showed that the treatment was generally tolerable and proved to have an antitumor activity.

The link between human epidermal growth factor receptor 2 (Her-2) and VEGF was reported to have a major role in predicting clinical outcome in primary breast cancer. ${ }^{24}$ Consequently, various combination therapies were assessed 
against these two proteins when treating breast cancers with Her-2 overexpression. A phase I study using a combination of bevacizumab and trastuzumab in nine patients was reported..$^{25}$ The reported results show 11\% CRs, 44\% PRs, and $22 \%$ SDs.

A number of trials of bevacizumab combined with other anticancer compounds were assigned in patients with metastatic breast cancer (MBC). ${ }^{26}$ This report discussed the combination of bevacizumab and chemotherapy including docetaxel which is known for its synergistic suppression of capillary vessel formation. It also discussed a study where 21 patients with inflammatory and locally advanced breast cancer (LABC) were assigned a combination therapy of bevacizumab and doxorubicin. This particular report showed $67 \%$ PRs, 24\% SDs, and 9\% PDs. Additional phase II trial was conducted in 49 patients to assess the vascular effects on tumor regression when using a monotherapy with docetaxel as opposed to a combination therapy of bevacizumab and docetaxel during the treatment of LABC. This study reported 14.3\% CRs, 65.3\% PRs, and 10\% disease progression. A phase III study of a monotherapy with capecitabine and a combination of capecitabine and bevacizumab was conducted in 462 patients with MBC. ${ }^{27}$ This report showed that the combination therapy increased the objective response rate from $9.1 \%$ to $19.8 \%$ as opposed to the monotherapy.

\section{A pre-plan for an anticancer three-drug compound design}

A specific dose of dual ErbB1/ErbB2 inhibitor lapatinib (GW572016) was needed to induce poly(adenosine diphosphate-ribose) polymerase (PARP) cleavage and DNA fragmentation to inhibit activation of EGFR and ErbB2 as well as the downstream MAPK and AKT pathways. ${ }^{28}$ Various experiments validated that a concentration higher than the inhibitory concentration (IC) $)_{50}$ determines the induction of apoptosis. Conversely, small inhibitory effects on EGFR or ErbB2 phosphorylation in tumor cells were observed when concentrations are below the $\mathrm{IC}_{50}$ value. ${ }^{29}$

Furthermore, it was reported that for some chronic, physiological diseases, synergism or antagonism at low concentration level also contribute towards treatment. ${ }^{30}$ However, for infectious diseases or cancer therapies, synergism at high effect levels including equilibrium dialysis (ED) ${ }_{90}, \mathrm{ED}_{95}$, or $\mathrm{ED}_{99}$, are considered to be more therapeutically appropriate as opposed to low effect levels such as $\mathrm{ED}_{30}$ or $\mathrm{ED}_{50}$.

The number of events, which occur during the process of killing cancer cells through the combination of two cytotoxic agents, is unknown. Evaluating synergism or antagonism and the explanation of how or why it happens represents different issues. ${ }^{30}$ Using the latest technological tools, it is possible to design the primary, secondary, and tertiary structure of an enzyme or a receptor as depicted in Figure $2,{ }^{8}$ however, it is still hard to design an inhibitor for the drug development process. In addition, the prediction of synergism or antagonism for enzymes or receptors is even more complex.

A pre-plan for conducting a three-drug combination therapy helps to seamlessly analyze two-drug compounds before reaching the three-drug compound. To demonstrate this scenario, I propose an experiment to design an anticancer threedrug combination composed of the following compounds: (1) an HDAC inhibitor "HDAC/INH”, (2) a VEGF receptor kinase inhibitor "VEGFR/INH", and (3) an EGFR/ERbB2 inhibitor "EGFR/ERbB2/INH". Figure 3 illustrates the design of the proposed experiment, in which each inhibitor refers to a drug. This proposal includes a set of three two-drug compounds, one three-drug compound, and a set of three three-drug compounds given as two-drug compounds consecutively.

In this experiment, it is proposed to use three drugs as two drugs as they are represented within square brackets in Figure 3. The benefit of this process is to gather valuable information when combining two drugs, which can be vital in spawning a three-drug compound. It embodies a process to generate novel compounds that incorporates the cytotoxicity factors of both compounds, while it helps to identify the most valuable candidate that satisfies the constraints of preclinical models. This process also helps to bridge the gap between preclinical models and clinical outcome through accurate prediction of toxicity levels.

\section{Challenges in translating experimental studies into clinical trials}

The drug development process is characterized by being highrisk, long-term, and costly. From a pool of 5,000 compound candidates entering the first development phase, only 5 will reach clinical testing. ${ }^{31}$ Furthermore, a new drug enters phase 1 testing, only after it endures at least 10 years of preclinical experimentation. It has been reported that in the USA, the population of adult cancer patients who are eligible to participate in clinical trials is between $2 \%$ and $3 \%$. Since the resources are very limited, drug sponsors search for cases from other parts of the world. ${ }^{32}$

Novel drug developments need to use an improved method to define the optimal biological dose rather than the maximum tolerated dose. These methods and studies need 


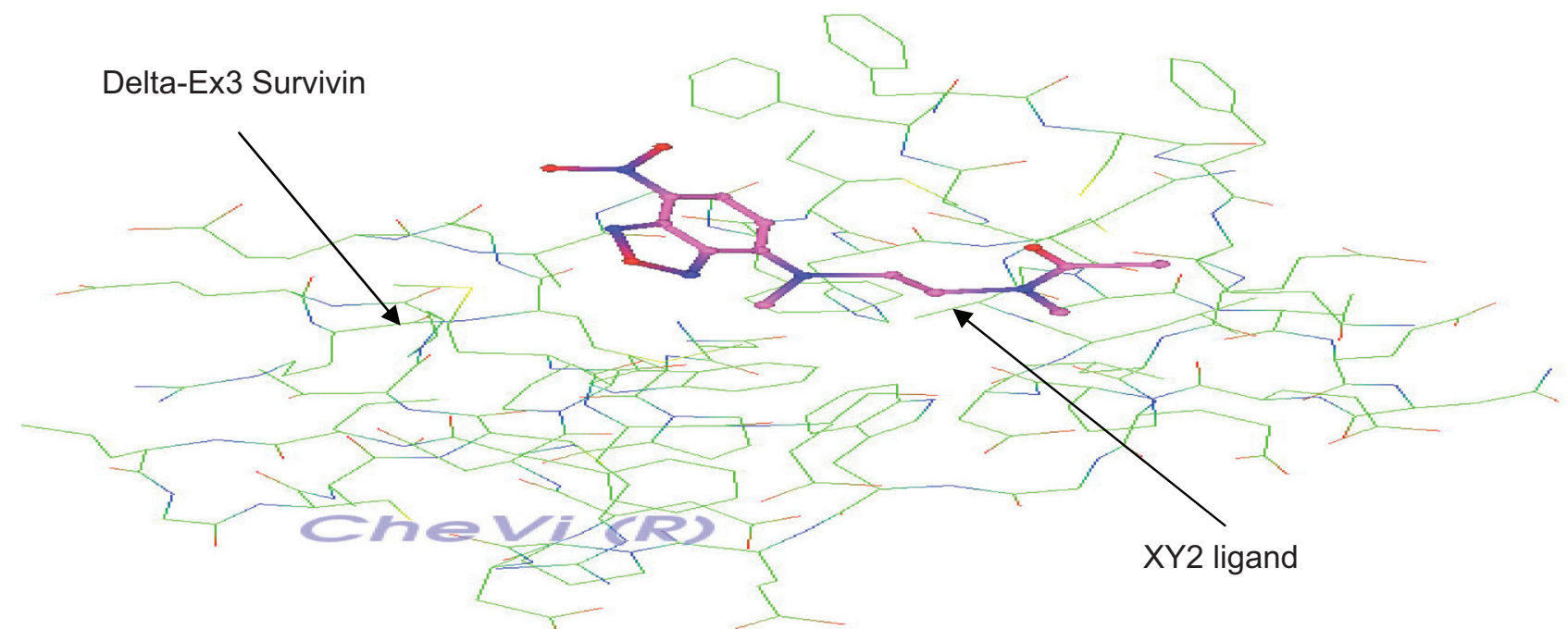

Figure 2 Complex receptor-ligand: Delta-Ex3 (a Survivin isoform) and XY2 (an HRas ligand). This complex has been properly visualized using CheVi (R) (a Linux-based Chemical Visualizer), where XY2 ligand is shown docked along Delta-Ex3's surface and deeply embedded in a particular active site.

to consider additional factors such as pharmacokinetics and pharmacodynamics of the compound instead of merely tumor size. The design of preclinical models based on these considerations and others such as appropriate endpoints and surrogate markers of response will lead to prospective clinical trials. This will allow preclinical models to predict clinical outcome with an adequate accuracy.

There are numerous challenges in translating experimental findings into clinical trials including a growing need to identify improved biomarkers that accurately predict the risk of the compound-related toxicity. This is associated with the quality of the preclinical models in predicting the corresponding toxicity. Hence, the use of nonclinical systems including animals, tissues slices, cell cultures, and in silico models, will not always help to accurately predict toxicity levels in humans. Human tumor xenografts grown subcutaneously have been used in preclinical testing when performed in immune deficient mice. ${ }^{33}$ These models were successful

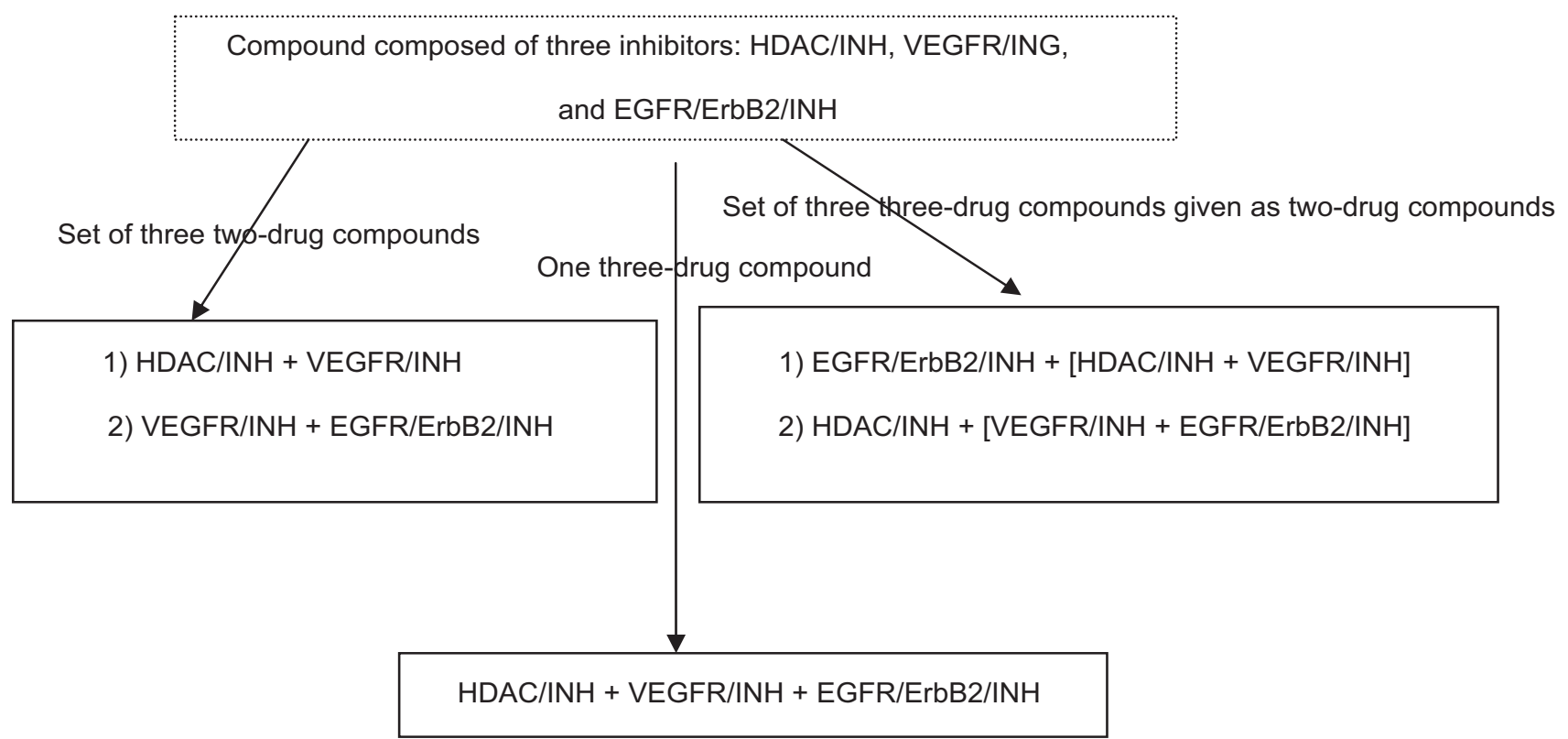

Figure 3 Design of the proposed three-drug combination experiment.

Abbreviations: EGFR, epidermal growth factor receptor; ErbB2, epidermal receptor growth factor 2; HDAC, histone deacetylase; INH, inhibitor; VEGF, vascular endothelial growth factor. 
in cytotoxic agents. ${ }^{34}$ However, the validity of these kinds of experiments did not reach the expected predictive indicator of clinical activity except when pharmacokinetically clinically equivalent drug doses are tested. ${ }^{35}$ Biomarkers that measure the molecular changes that lead to toxicity are considered to provide a useful early detection of toxicity. This type of biomarkers will help investigators to conduct a predictable and successful transition from preclinical studies to clinical trials.

\section{Conclusion}

This paper describes an anticancer drug design that combines three types of drugs as well as the challenges that lay ahead in setting up this type of experiment. The proposed threedrug compound warrants further experiments and clinical tests to demonstrate its candidacy among anticancer drugs. An analysis of toxicity is also required to be conducted on the newly designed compound. This analysis includes whether any two drugs, within the thee-drug compound, have overlapping or nonoverlapping toxicity, which includes gastrointestinal toxicity, cardiotoxicity, renal toxicity, and neurotoxicity. However, the generation of newly designed compounds with nonoverlapping toxicities may not be valuable since this will increase treatment costs and decrease patient compliance.

\section{Disclosure}

The author reports no conflict of interest in this work.

\section{References}

1. Esteller M. Cancer epigenomics. Nat Rev Genet. 2007;8:286-298.

2. Jones PA, Baylin SB. The epigenomics of cancer. Cell. 2007;128: 683-692.

3. Yoo CB, Jones PA. Epigenetic therapy of cancer. Nat Rev Drug Disc. 2006;5:37-50

4. Qian DZ, Wang X, Kachhap SK, et al. The histone deacetylase inhibitor NVP-LAQ824 inhibits angiogenesis and has a greater antitumor effect in combination with the vascular endothelial growth factor receptor tyrosine kinase inhibitor PTK787/ZK222584. Cancer Res. 2004;64:6626-6634.

5. Venkateswarlu S, Dawson DM, St Clair P, et al. Autocrine heregulin generates growth factor independence and blocks apoptosis in colon cancer cells. Oncogene. 2002;21:78-86.

6. Jackson JG, St Clair P, Sliwkowski MX, et al. Blockade of epidermal growth factor- or heregulin- dependent ErbB2 activation with the anti-ErbB2 monoclonal antibody $2 \mathrm{C} 4$ has divergent downstream signaling and growth effects. Cancer Res. 2004;64:2601-2609.

7. Gasparini G, Longo R, Fanelli M, et al. Combination of antiangiogenic therapy with other anticancer therapies: results, challenges, and open questions. J Clin Oncol. 2005;1295-1311.

8. Ezziane Z. Molecular docking and analysis of survivin delta-ex3 isoform protein. The Open Medicinal Chemistry Journal. 2008;2:16-20.

9. von Mehren M, Schilder RJ, Cheng JD, et al. A phase I study of the safety and pharmacokinetics of trabectedin in combination with pegylated liposomal doxorubicin in patients with advanced malignancies. Ann Oncol. 2008;19(10):1802-1809.
10. Sabnani I, Zucker MJ, Rosenstein ED, et al. A novel therapeutic approach to the treatment of scleroderma-associated pulmonary complications: safety and efficacy of combination therapy with imatinib and cyclophosphamide. Rheumatology. 2009;48(1):49-52.

11. O'Byrne KJ, Danson S, Dunlop D, et al. Combination therapy with gefitinib and rofecoxib in patients with platinum-pretreated relapsed non-small-cell lung cancer. J Clin Oncol. 2007;25(22):3266-3273.

12. Konstas AGP, Mikropoulos D, Dimopoulos AT, et al. Second-line therapy with dorzolamide/timolol or latanoprost/timolol fixed combination versus adding dorzolamide/timolol fixed combination to latanoprost monotherapy. Br J Ophthalmol. 2008;92:1498-1502.

13. Sherwood MB, Craven ER, Chon C, et al. Twice-daily $0.2 \%$ brimonidine $-0.5 \%$ timolol fixed-combination therapy vs monotherapy with timolol or brimonidine in patients with glaucoma or ocular hypertension: A 12-month randomized trial for the Combigan Study Groups I and II. Arch Ophthalmol. 2006;124:1230-1238.

14. Braiteh F, Soriano AO, Garcia-Manero G, et al. Phase I study of epigenetic modulation with 5-azacytidine and valproic acid in patients with advanced cancers. Clin Can Res. 2008;14:6296-6301.

15. Candelaria M, Gallardo-Rincón D, Arce C, et al. A phase II study of epigenetic therapy with hydralazine and magnesium valproate to overcome chemotherapy resistance in refractory solid tumors. Ann Oncol. 2007;18(9):1529-1538.

16. Schrump DS, Fischette MR, Nguyen DM, et al. Clinical and molecular responses in lung cancer patients receiving romidepsin. Clin Can Res. 2008;14:188-198.

17. Berenson JR, Yang HH, Sadler K, et al. Phase I/II trial assessing bortezomib and melphalan combination therapy for the treatment of patients with relapsed or refractory multiple myeloma. J Clin Oncol. 2006;24(6):937-944.

18. Berenson JR, Matous J, Swift RA, et al. Phase I/II study of arsenic trioxide/bortezomib/ascorbic acid combination therapy for the treatment of relapsed or refractory multiple myeloma. Clin Can Res. 2007;13:1762-1768.

19. Goldstein BJ, Feinglos MN, Lunceford JK, et al. Effect of initial combination therapy with sitagliptin, a dipeptidyl peptidase- 4 inhibitor, and metformin on glycemic control in patients with type 2 diabetes. Diabetes Care. 2007;30:1979-1987.

20. Rosenstock J, Sugimoto D, Strange P, et al. Insulin glargine or rosiglitazone added to combination therapy of sulfonylurea plus metformin in insulin-naïve patients. Diabetes Care. 2006;29:554-559.

21. Dy GK, Bruzek LM, Croghan GA, et al. A phase I trial of the novel farnesyl protein transferase inhibitor, BMS-214662, in combination with paclitaxel and carboplatin in patients with advanced cancer. Clin Can Res. 2005;11:1877-1883.

22. Diéras V, Bonneterre J, Laurence V, et al. Phase I combining a p-glycoprotein inhibitor, MS209, in combination with docetaxel in patients with advanced malignancies. Clin Can Res. 2005;11:6256-6260.

23. Forster M, Kaye S, Oza A, et al. A Phase Ib and pharmacokinetic trial of patupilone combined with carboplatin in patients with advanced cancer. Clin Can Res. 2007;13:4178-4184.

24. Konecny GE, Pegram M, Venkatesan NM, et al. Activity of the dual kinase inhibitor lapatinib (GW572016) against HER-2 overexpressing and trastuzumab-treated breast cancer cells. Cancer Res. 2006;66(3):1630-1639.

25. Pegram M, Yeon C, Ku N, et al. Phase I combined biological therapy of breast cancer using two humanized monoclonal antibodies directed against HER-2 protooncogene and vascular endothelial growth factor (VEGF) Proc. SABCS 2004 [abstract 3039]. Breast Cancer Res Treat. 2005;90(2):193-214.

26. Milano A, Nasti G, Laffaioli RV, et al. First line targeted therapies in breast cancer: focus on bevacizumab. Biologics: Targets and Therapy. 2007;1(1):3-10.

27. Miller KD, Chap LI, Holmes FA, et al. Randomized phase III trial of capecitabine compared with bevacizumab plus capecitabine in patients with previously treated metastatic breast cancer. J Clin Oncol. 2005a;23:792a-799a. 
28. Zhou Y, Song L, Hu Yi P, et al. Blockade of EGFR and ErbB2 by the novel dual EGFR and ErbB2 tyrosine kinase inhibitor GW572016 sensitizes human colon carcinoma GEO cells to apoptosis. Cancer Res. 2006;66(1):404-411.

29. Rusnak DW, Lackey K, Affleck K, et al. The effects of the novel, reversible epidermal growth factor receptor/ErbB-2 tyrosine kinase inhibitor, GW2016, on the growth of human normal and tumor-derived cell lines in vitro and in vivo. Mol Cancer Ther. 2001;1(2):85-94.

30. Chou TC. Theoretical basis, experimental design, and computerized simulation of synergism and antagonism in drug combination studies. Pharmacolo Rev. 2006;58:621-681.

31. Tufts Center for the Study of Drug Development. Recent news from the Tufts Center for the Study of Drug Development. 2001. [Cited Jan 10, 2009]. Available from: http://csdd.tufts.edu/NewsEvents.
32. Schein PS, Scheffler B. Barriers to efficient development of cancer therapeutics. Clin Can Res. 2006;12(11):3243-3248.

33. Newell P, Villanueva A, Friedman SL, et al. Experimental models of hepatocellular carcinoma. $J$ Hepatol. 2008;48:858-879.

34. Huynh H, Soo KC, Chow PK, et al. Xenografts of human hepatocellular carcinoma: a useful model for testing drugs. Clin Can Res. 2006;12:4306-4314

35. Kerbel RS. Human tumor xenografts as predictive preclinical models for anticancer drug activity in humans: better than commonly perceived-but they can be improved. Cancer Biol Ther. 2003;2:S134-S139. 
